\title{
La edad y el rendimiento académico universitario Age and Academic Performance of College Students
}

\author{
Portillo-Tarragona, Pilar ${ }^{1}$, Marín-Vinuesa, Luz María ${ }^{2}$, Scarpellini, Sabina ${ }^{1}$ \\ portillo@unizar.es, luz-maria.marin@unirioja.es, sabina@unizar.es \\ ${ }^{1}$ Departamento de Contabilidad y Finanzas \\ Universidad de Zaragoza \\ Zaragoza, España \\ ${ }^{2}$ Departamento Economía y Empresa \\ Universidad de La Rioja \\ Logroño, España
}

\begin{abstract}
Resumen- En este trabajo se analiza la relación entre la edad y el rendimiento académico universitario en un curso de adaptación al Grado en Administración y Dirección de Empresas que por su naturaleza, dirigido a titulados de la anterior ordenación en España, posibilita la creación de grupos de población heterogénea. Para ello. se prepara y analiza una base de datos de 72 estudiantes matriculados en una asignatura de finanzas del curso de adaptación. Los resultados del análisis de regresión muestran que el incremento en la edad afecta negativamente al rendimiento del estudiante, en la primera convocatoria del semestre en el que se ubica la asignatura.. Además se comprueba que los efectos de la edad sobre el rendimientoacadémico universitario son independientes del sistema de evaluación.
\end{abstract}

Palabras clave: Rendimiento académico universitario, edad, sistema de evaluación continuada, curso de adaptación, educación superior.

Abstract- This paper analyzes the relationship between the age and the academic performance of college students. The empirical study is carried on a group of students who were following the Adjustment Course to the Degree in Business Administration and Management in a Spanish public university; this Course is designed for graduates of the previous management and enables the creation of heterogeneous population groups. To this end, a database of 72 students enrolled in a course of finance of the adaptation course is prepared and analyzed. Results of regression analysis show the negative effects of the age on students' academic performance in the first round of the semester in which the degree course is located. Moreover, effects of age on students' academic performance are not different from Students who choose a continuous assessment system and students who choose a comprehensive evaluation system.

Keywords: Academic performance, Student' Age, Continuous Assessment System, Adjustment Course to the Degree, Higher Education

\section{INTRODUCCIÓN}

Los constantes cambios en las circunstancias económicas, sociales y tecnológicas unido al propio proceso emprendido por las universidades europeas para la construcción del Espacio Europeo de Educación Superior (EEES) hace que el paso por la universidad sea contemplado no sólo por estudiantes con edades teóricas de acceso, es decir, que han seguido ininterrumpidamente el itinerario temporal e institucional del proceso educativo hasta incorporarse al ámbito universitario, sino por estudiantes de mayor edad, que desean progresar en el sistema educativo bien procedentes de ordenaciones anteriores y con experiencia laboral, o bien desean iniciar, concluir, adaptar o ampliar sus estudios universitarios.

De este modo el abanico de características entre los estudiantes universitarios en las nuevas titulaciones se amplía, como se indica en Morgan (2012). Los trabajos de Schuezte y Slowey (2002), Leder y Forgasz (2004) y Ramsay et al. (2007) señalan que las diferentes edades, distintas procedencias o la experiencia previa de acceso provocan un incremento de la diversidad en la educación superior. En este contexto de diversidad, cobra un interés especial el estudio de las relaciones entre la edad de los estudiantes y su rendimiento académico universitario.

Hasta el momento, las investigaciones sobre los efectos de la edad sobre el rendimiento académico universitario arrojan conclusiones contradictorias. En general se observa que al trabajar con muestras correspondientes a programas de enseñanza presencial, los escenarios de análisis presentan poblaciones homogéneas en edad, en las que mayoritariamente suele coincidir con la teórica de acceso (Martínez y Galán, 2000). Por otra parte, los trabajos donde se analizan muestras más heterogéneas respecto a la edad (Cheung y Kan, 2002), en general son estudios referidos a programas de enseñanza a distancia y no presencial, en los que se ofrece cierta flexibilidad a los estudiantes para que puedan adaptar la estructura a sus propias necesidades. El presente trabajo pretende cubrir este vacío, analizando cómo afecta el incremento de edad del estudiante al rendimiento académico universitario sobre una muestra heterogénea de estudiantes que cursan una asignatura presencial.

La elección de una asignatura de carácter presencial para realizar este trabajo nos parece interesante por dos razones. Primero, porque nos permite estudiar variables tales como la asistencia a clase y el seguimiento y control de diferentes actividades presenciales, encaminadas a la autorregulación del aprendizaje dentro de un sistema de evaluación continuada. Dichas variables son relevantes en el estudio de las relaciones entre la edad y el rendimiento académico. Respecto a la asistencia a clase, en los estudios de Cheung y Kan (2002) y Tejedor (2003), se encuentra una relación positiva entre la asistencia y el rendimiento. Asimismo, la incidencia del sistema de evaluación sobre el rendimiento final del estudiante se analiza en los trabajos que valoran la utilización de diferentes sistemas y herramientas de evaluación, como en Clavería (2009) y Fraile et al. (2013), en los que se observa un efecto positivo cuando se utilizan sistemas de evaluación continuada. En segundo lugar, la aplicación de nuestro estudio 
a un curso presencial nos permite avanzar en la línea descrita, determinando si los posibles efectos de la edad sobre el rendimiento académico universitario son independientes del sistema utilizado en la evaluación de la asignatura.

Para realizar el estudio tomamos como referencia una asignatura de finanzas, de carácter presencial de un curso de adaptación al Grado en Administración y Dirección de Empresas (ADE), ofertado por un centro universitario español, adscrito a la Rama de Ciencias Sociales que, por su naturaleza, dirigido a titulados de la anterior ordenación, posibilita la creación de grupos de población heterogénea. En este sentido, siguiendo a Tejedor (2003), por rendimiento universitario entendemos el nivel de conocimientos que ha demostrado el estudiante a lo largo del proceso de evaluación de la asignatura y que de modo operativo se mide a partir de la calificación obtenida por el estudiante.

Sobre dicha muestra de estudiantes, heterogénea respecto a la edad y la experiencia, en este trabajo analizamos, en primer lugar, si el incremento en la edad del estudiante afecta a su rendimiento académico universitario. Y, en segundo lugar, si dicha relación se observa independientemente del sistema de evaluación llevado a cabo. Así, los resultados de la aplicación empírica que presentamos permiten avanzar en la línea de estudio de las relaciones entre la edad y el rendimiento académico universitario, incrementando el conocimiento de factores que son importantes en la oferta académica de las materias de los cursos de adaptación.

\section{CONTEXTO}

La edad del estudiante es uno de los muchos factores que pueden influir en su rendimiento académico universitario. La teoría de la Elección Racional (Allingham, 2002), relacionada con la Teoría del Capital Humano (Becker, 1964), explicaría el interés de los estudiantes, pertenecientes a un segmento de edad distinto al teórico de acceso, por su incorporación a un curso de adaptación a un grado universitario, en la medida que los beneficios esperados tras su superación (mejores oportunidades laborales) rebasarían los costes asociados al mismo (obligaciones o cargas asociadas a la nueva dedicación). La relación que un perfil como el descrito tendría con el rendimiento académico universitario podría ampararse en la Teoría de retención estudiantil (Tinto, 1975), puesto que la transición del ambiente laboral hacia el universitario resulta más difícil cuanto mayor es la diferencia generacional favoreciendo el abandono y justificando la relación con un peor rendimiento académico universitario.

Tradicionalmente la madurez, el espíritu crítico, la perseverancia y la experiencia que lleva implícita una mayor edad, frente a estudiantes jóvenes, podrían sugerir que la edad se relacione positivamente con determinados estilos de aprendizaje (Justice y Dornan, 2001, McKenzie y Gow, 2004) y por extensión, con el rendimiento académico universitario. Sin embargo, las cargas económicas, laborales y familiares (Tones et al., 2009), la distancia temporal de los estudios previos de acceso (Peiperl y Trevelyan, 1997) o el déficit de habilidades tecnológicas, que pueden facilitar el aprendizaje (Tones et al., 2009), que generalmente acompañarían a estos perfiles, también se han identificado como razones de peso que no favorecen la mejora del rendimiento académico universitario.
Sobre la base de estas razones no favorecedoras de la mejora del rendimiento académico universitario, una gran parte de estudios previos constatan la existencia de relaciones negativas entre la edad y el rendimiento. En el trabajo de Peiperl y Trevelyan (1997), implementado en el contexto de MBA, se sugiere una relación inversa entre la edad del estudiante y su rendimiento, señalando como explicación la mayor proximidad temporal de los estudiantes más jóvenes a un entorno académico, lo que contribuiría a conseguir un mejor desempeño en dicho ámbito, frente a perfiles con una mayor experiencia laboral y mayor edad. También se constata una relación negativa en los trabajos de Jiménez-Fernández (1987) y Cortés y Palomar (2008). El estudio de Cortés y Palomar (2008) se centra en estudiantes de nuevo ingreso en una licenciatura, en la que se muestra una edad muy próxima a la teórica de acceso, encontrando mayores puntuaciones en los estudiantes de menor edad. En Jiménez-Fernández (1987) se analizan distintos grupos de estudiantes de todo el abanico de titulaciones que ofrece un centro adscrito a una institución universitaria de educación a distancia, en los que se observa dispersión en la variable edad, también se corrobora la relación negativa entre la edad y el rendimiento académico universitario.

Otros estudios han concluido que las relaciones entre la edad y el rendimiento académico universitario, no presentan resultados en el mismo sentido. En Cheung y Kan (2002), Byrne y Flood (2008) no se encuentra influencia de la edad sobre el rendimiento, mientras que Porto y Di Gresia (2004) y Guney (2009) encuentran una relación positiva. De la misma forma que en Jiménez-Fernández (1987), en el estudio de Cheung y Kan (2002) se trabaja con grupos en los que se observa dispersión en la variable edad, y que se corresponden con enseñanzas a distancia, pero en este último el estudio se centra en una asignatura de contabilidad de gestión, en el que la mayor parte de los estudiantes lo compatibilizan con su trabajo, incluso los estudiantes muy jóvenes, caracterizados por una elevada motivación académica, por lo que no se constata ese efecto negativo. El hecho de no presentar un planteamiento presencial facilitaría su compatibilización con otras cargas inherentes a la edad. Por otra parte, en Guney (2009) la relación positiva se justifica por un comportamiento más disciplinado, inherente a los estudiantes de mayor edad, mientras que en Porto y Di Gresia (2004), se comprueba una relación positiva pero vinculada a curso académico, conforme se va avanzando en cursos sucesivos el rendimiento mejora. Si la variable edad se estudia dentro del mismo curso (por ejemplo primer curso) y con grupos homogéneos tendría un efecto negativo, pues se asocia a que esa diferencia, frente a la teórica de acceso, se debe a fracasos académicos previos. Por el contrario cuando la variable edad se asocia a curso, aquella se asocia a la madurez del estudiante (Tejedor, 2003).

Parece obvio pensar que la diferencia de edad entre grupos de estudiantes, con distinta experiencia laboral, puede resultar ventajosa tanto en la dinámica del desarrollo de la clase como en el proceso de enseñanza aprendizaje, debido a la aportación de distintas experiencias previas por parte de cada estudiante. Sin embargo, cuando esa diferencia de edad supera determinados periodos de tiempo, la experiencia previa puede no beneficiar al rendimiento individual de cada estudiante; así autores como Peiperl y Trevelyan (1997) sugieren que ante niveles de diferencia de edad de cuatro o cinco años, podrían observarse efectos negativos. Sobre la base de este razonamiento, y teniendo en cuenta lo expuesto anteriormente, 
en la muestra de estudiantes del curso de adaptación, caracterizada por su heterogeneidad respecto a la edad, ya que se trata de grupos de edad alejados de la teórica de finalización de estudios previos, y también respecto a la experiencia laboral afín a la titulación, entendemos que el incremento en la edad del estudiante, unido a las cargas asociadas (laborales, familiares y financieras), podría afectar negativamente al rendimiento del estudiante. En este sentido plantemos la primera hipótesis del estudio: H1: El incremento en la edad del estudiante afecta negativamente al rendimiento académico en el curso de adaptación al grado.

Por otra parte, se admite la relación entre el sistema de evaluación utilizado y el rendimiento académico universitario final del estudiante (Coll et al., 2007, Clavería, 2009 y Fraile et al., 2013), admitiéndose los efectos positivos que sobre el rendimiento ejercen los sistemas de evaluación continuada. El sistema de evaluación continuada, frente a la evaluación tradicional, permite, por un lado, realizar un seguimiento de los resultados de aprendizaje del estudiante facilitando la introducción de medidas adecuadas para corregir las situaciones observadas (Coll et al. 2007). En este sentido, en Fraile et al. (2013) se comparan sistemas tradicionales y de evaluación continuada y se corrobora que la mejora pedagógica conseguida con evaluación continuada termina reflejándose en una mejora de la calificación final. Daugherty et al. (2015) señalan que el establecimiento de pruebas intermedias permite al profesorado identificar a los estudiantes con problemas y a los estudiantes afectados tomar conciencia de su situación. De manera que los resultados intermedios tienen un papel formativo para el estudiante (Martínez, 2013).

Además, la elección por parte del estudiante de un sistema de evaluación integrado por diferentes pruebas y actividades, todas ellas supervisadas por el profesor con y sin repercusión en la calificación final, es el reflejo de su predisposición positiva a la hora de enfocar el estudio y de su interés por un feedback que le permita ir adoptando mejoras en su aprendizaje (Clavería, 2009). Partiendo de la base de que esta predisposición positiva debería observarse en el estudiante universitario, independientemente de su edad de acceso a la titulación, nos parece interesante analizar si la relación entre el incremento de edad y el rendimiento académico universitario del estudiante es independiente del sistema de evaluación llevado a cabo, o si por el contrario el hecho de optar a un sistema de evaluación continuada, frente a un sistema global de evaluación, podría afectar a la relación anterior atenuando los efectos planteados en la primera hipótesis del estudio.

Bajo nuestro planteamiento inicial, la relación entre el incremento de edad y el rendimiento académico universitario del estudiante debería mantenerse independiente del sistema de evaluación llevado a cabo. En ese sentido, planteamos la segunda hipótesis del estudio: H2: la relación entre el incremento en la edad $\mathrm{y}$ el rendimiento académico universitario es independiente del sistema de evaluación adoptado en el curso de adaptación al grado.

\section{DESCRIPCIÓN}

En España diversas normas propician el inicio del proceso de cambio y posterior reestructuración de las enseñanzas universitarias. La Ley Orgánica 4/2007, de 12 de abril, de Universidades, regulada por el Real Decreto 1393/2007, de 29 de octubre, modificado por el Real Decreto 861/2010 de 2 de julio, establecen la ordenación de las enseñanzas universitarias oficiales, cuya organización, en nuevas titulaciones, potencian la apertura y movilidad de los estudiantes a nivel nacional e internacional, ampliando el ámbito en el que pueden operar las nuevas titulaciones entre los países que forman parte del EEES, con las consiguientes repercusiones profesionales.

De este modo el título de Grado además de permitir mejorar el currículum de las personas que están en edad de trabajar, flexibiliza y amplía el territorio en el que puede ser utilizado, por lo que resulta de interés, no sólo para los estudiantes de planes correspondientes a las anteriores titulaciones, de primer y segundo ciclo, sino para los ya titulados, de planes anteriores, interesados en adaptar su antigua titulación.

La oferta de grados con curso de adaptación se dirige a las titulaciones de Diplomado, Ingeniero Técnico o Arquitecto Técnico de la anterior ordenación. Según información de la Agencia Nacional de Evaluación de la Calidad y Acreditación (ANECA), la mayoría de los referidos cursos se encuentran adscritos fundamentalmente a dos ramas de conocimiento, la rama de Ciencias Sociales y Jurídicas e Ingeniería y la rama Arquitectura, y en menor medida a la de Ciencias de la Salud.

El presente estudio comprende a estudiantes matriculados en una asignatura de finanzas, de carácter presencial, impartida durante el primer semestre del curso de adaptación para acceder al Grado en ADE. Para realizar el análisis se utiliza información del estudiante sobre el rendimiento académico universitario, la edad y otra información académica y de historial de acceso. La muestra está constituida por 72 estudiantes. Su composición respecto al sexo de los estudiantes es muy similar (48,6\% hombres y 51,4\% mujeres). Respecto al domicilio habitual, la mayoría de estudiantes residen en la misma localidad $(70,8 \%)$ y, por cercanía, los estudiantes que residen en un barrio rural, en la provincia y en la propia comunidad autónoma representan un $8,3 \%$, un $6,9 \%$ y un $11,1 \%$, respectivamente; los residentes en otra comunidad autónoma representan una participación muy escasa (2,8\%).

El curso de adaptación tiene como objetivo que los titulados de la anterior ordenación puedan adquirir los complementos formativos para ser equiparados con las nuevas titulaciones, siendo su organización responsabilidad de la universidad oferente. La universidad encargada de su oferta, a efectos de su organización prevé la adjudicación de plazas, cuando la demanda supere a la oferta, se realizará sobre la base de los criterios del expediente de acceso de su anterior titulación (valorado hasta 10 puntos) y de la experiencia laboral acreditada y valorada (otorgando hasta 5 puntos con un máximo de 20 años de trabajo acreditado). Dicho sistema se mantiene hasta adjudicar el total de las plazas ofertadas en los sucesivos llamamientos.

El llamamiento en el que resultan admitidos los estudiantes condiciona la fecha de incorporación al curso (mayor sensibilidad en las asignaturas de primer semestre) así como el perfil de edad y experiencia del grupo (los llamamientos posteriores se suelen corresponder con perfiles de menor edad y experiencia). En la muestra, la mayoría de los estudiantes (80,6\%) acceden sin llamamiento previo, frente a un 13,9\% de estudiantes que acceden en el primer llamamiento, un $4,2 \%$ en el segundo y un $1,4 \%$ en el tercero de ellos.

El curso de adaptación se imparte en horario de tarde y los estudiantes se deben matricular en un total de 36ECTS correspondientes a seis asignaturas, entre las que se incluye un 
Trabajo Fin de Grado, además de 2ECTS correspondientes a la asignatura de Idioma nivel B1. Además se imparte en modalidad presencial y aunque la asistencia no es obligatoria, por disposición normativa de la universidad, en la asignatura se recomienda la asistencia a clase con el fin de facilitar el aprendizaje de la materia. Respecto a la asistencia de los estudiantes a los controles aleatorios, la mayoría de los estudiantes (52\%) asistió a los dos controles realizados, un $29,2 \%$ asistió sólo a uno de los controles y un 18,1\% de estudiantes no asistieron a ninguno de los dos.

Para la superación de la asignatura el estudiante puede optar por un sistema de evaluación continuada o un sistema de evaluación global. El primer sistema evalúa la adquisición de resultados de aprendizaje y el desarrollo de competencias por dos vías: a partir de la resolución de cuatro casos prácticos propuestos sobre determinadas áreas del programa, en el transcurso de las sesiones habilitadas para trabajo en grupos reducidos, y a partir de un examen final escrito. Si bien la puntuación máxima del conjunto de los casos representa un $20 \%$ de la nota final, el $80 \%$ restante corresponde a la nota alcanzada en el examen escrito de la correspondiente convocatoria oficial. Por otra parte, en el sistema de evaluación global, el $100 \%$ de la calificación se corresponde con la puntuación alcanzada en el examen escrito de la correspondiente convocatoria oficial. Respecto a la opción del sistema de evaluación, la mayoría de los estudiantes de la muestra $(69,4 \%)$ ha optado por un sistema de evaluación continuada, frente a un 30,6\% de estudiantes que expreso su interés por el sistema de evaluación global.

Independientemente del sistema de evaluación elegido y con la finalidad de realizar un seguimiento de los resultados de aprendizaje que se van alcanzando, a mitad del semestre se realiza una prueba a modo de ensayo intermedio, de carácter voluntario, cuyo resultado no puntúa en la calificación final. El formato y características de dicha prueba son similares a los del examen de la convocatoria oficial, lo que servirá para detectar puntos críticos en el proceso de enseñanza aprendizaje. En nuestro caso, todos los estudiantes de la muestra realizaron la prueba voluntariamente.

\section{A. Variables utilizadas en el análisis}

Rendimiento académico universitario (RAU). Esta variable se ha aproximado para la primera convocatoria del curso, convocatoria de febrero, cuantificándose con la calificación final conseguida por el estudiante en dicha convocatoria. Si bien el rendimiento, a efectos operativos, lo vamos a considerar desde un punto de vista inmediato y estricto, medido por la presentación y éxito alcanzado en la calificación de la asignatura, no es menos cierto que éste tiene repercusiones en un sentido más amplio (Tejedor y GarcíaValcárcel, 2007), ya que condiciona la finalización de los estudios en un determinado período y en última instancia del abandono de los mismos.

Edad (E). Variable demográfica cuya información es extraída a partir de la información de las fichas de los estudiantes.

Sistema de evaluación continuada (EC). Variable académica que se incluye como variable dummy que toma el valor 1 para reflejar la opción al sistema de evaluación continuada por parte del estudiante y el valor 0 en otro caso.

Llamamiento (Ll). El llamamiento en el que resultan admitidos los estudiantes (primero/segundo/tercero) condiciona la fecha de incorporación al curso. Se corresponde con una variable de historial de acceso: nota del expediente de acceso, correspondiente a su anterior titulación y a la experiencia laboral (valorada y acreditada); ambas publicadas en los listados de los respectivos llamamientos, en el tablón de anuncios del centro.

Asistencia a clase (A). La asistencia periódica a clase se ha medido con controles aleatorios de presencia en fechas en las que no estaban previstas pruebas de seguimiento del aprendizaje. Respecto a la asistencia clase o a tutorías por parte de los estudiantes, en las investigaciones de Cheung y Kan (2002) y Tejedor (2003), se encuentra una relación positiva entre la asistencia y el rendimiento.

Valoración del ensayo intermedio (V). Se ha aproximado con la calificación de la prueba de valoración intermedia. En la medida que replica, en formato reducido, el tipo de prueba con el que se enfrentarán en el examen final les permite reflexionar sobre el grado de consecución de los objetivos de aprendizaje alcanzados por el estudiante y reorientar, si fuera necesario, su preparación, por lo que es de esperar que la presentación a la misma mejore el rendimiento académico universitario final.

\section{B. Metodología}

Con objeto de dar respuesta a nuestra primera hipótesis de investigación, es decir, analizar si el incremento en la edad del estudiante afecta negativamente al rendimiento académico universitario en el curso de adaptación al grado, planteamos el modelo de regresión descrito en la ecuación (1):

$$
\mathrm{RAU} i=\beta \mathrm{o}+\beta 1 \mathrm{Ei}+\beta 2 \mathrm{EC}+\beta 3 \mathrm{Lli}+\beta 4 \mathrm{Ai}+\beta 5 \mathrm{Vi}+\mathrm{ei}
$$

Para dar respuesta a la segunda hipótesis, realizamos dos análisis. En primer lugar, analizamos si es posible contrastar un comportamiento significativamente distinto en la variable edad, respecto a los estudiantes que optaron al sistema de evaluación continuada y aquellos otros que no optaron. Para ello utilizamos el análisis ANOVA. En segundo lugar, con objeto de analizar si la influencia del incremento de edad en el rendimiento académico universitario es independiente, o no, del sistema de evaluación que el estudiante haya elegido, planteamos la ecuación (2). En dicha ecuación se incluye, además de las variables consideradas en la ecuación (1), una variable adicional generada a través del producto de la variable dummy (EC) con la variable edad.

\section{$\mathrm{RAU} i=\beta \mathrm{o}+\beta 1 \mathrm{Ei}+\beta 2 \mathrm{EC}-\beta^{\prime} 1\left(\mathrm{EC}^{*} \mathrm{E}\right) \mathrm{i}+\beta 3 \mathrm{Lli}+\beta 4 \mathrm{Ai}+\beta 5 \mathrm{Vi}+$ ei}

Para estimar los modelos de regresión se ha utilizado el método mínimo cuadrático ordinario (MCO). La salida del software informático empleado, STATA 12.0, nos ha proporcionado la estimación de los coeficientes de las variables incluidas en cada modelo. Además nos permite estudiar del ajuste y el cumplimento de las hipótesis estadísticas básicas del modelo.

\section{Resultados}

Los resultados de estimación del modelo (1) son robustos y muestran que tanto la edad del estudiante como la elección del sistema de evaluación continuada son factores explicativos del rendimiento académico universitario de los estudiantes del curso de adaptación. El modelo es altamente significativo de acuerdo con el test de significación global $(\mathrm{F}=15,17$; sig. 0,00) y el grado de ajuste alcanzado es bastante alto (R2 ajustado=0,53). El valor del factor de inflación de la varianza 
(VIF=1,26) indica la ausencia de multicolinealidad elevada entre las variables incluidas en el modelo. El valor del indicador Durbin-Watson (D-W=2,3) muestra la no correlación de las perturbaciones. La heteroscedasticidad no es un problema en los datos, tal como muestra el contraste de White ( $\chi 2$ de White=26,77; $\mathrm{p}=0,26$ ). El valor estimado del coeficiente de la variable E $(-0,06)$ muestra una relación negativa entre la edad y el rendimiento. Estos resultados no permiten rechazar la hipótesis 1 que defiende que el incremento en la edad del estudiante afecta negativamente al rendimiento académico universitario en el curso de adaptación al grado. Por otra parte, el valor estimado del coeficiente de la variable EC $(1,15)$ indica los efectos positivos del sistema de evaluación continuada sobre el rendimiento académico universitario.

Tabla 1. Resultados del modelo de regresión logística

\begin{tabular}{|c|c|c|c|c|}
\hline \multirow{2}{*}{ Variable } & \multicolumn{2}{|c|}{ Coeficientes } & \multirow{2}{*}{$\boldsymbol{*}$} & \multirow{2}{*}{ Sig. } \\
\cline { 2 - 3 } & Valor & Error & & 0,046 \\
\hline $\mathrm{E}$ & $-0,06$ & 0,03 & $-2,03$ & 03 \\
\hline $\mathrm{Ll}$ & $-0,45$ & 0,44 & $-1,02$ & 0,314 \\
\hline $\mathrm{A}$ & 0,94 & 0,40 & 2,33 & 0,023 \\
\hline $\mathrm{V}$ & 6,26 & 1,21 & 5,19 & 0,000 \\
\hline $\mathrm{EC}$ & 1,15 & 0,66 & 2,09 & 0,040 \\
\hline Constante & 3,10 & 1,08 & 2,87 & 0,006 \\
\hline
\end{tabular}

Previamente a la estimación del modelo (2) realizamos un ANOVA para comprobar la relación entre las variables: edad de los estudiantes (E) y opción al sistema de evaluación continuada (EC). Los resultados del análisis se recogen en la tabla 3. Para la variable edad no se observan diferencias estadísticamente significativas en sus valores medios en cada uno de los dos grupos que define la variable EC, esto es, en el grupo de estudiantes que opta al sistema de evaluación continuada y en el grupo de estudiantes que no eligen esta opción de evaluación. A la vista de estos resultados, no es posible afirmar la existencia de un comportamiento diferente para la variable edad entre los estudiantes que optaron al sistema de evaluación continuada y los que no optaron.

Siguiendo el esquema de trabajo propuesto estimamos el modelo de regresión (2). Respecto a los coeficientes de estimación, los resultados muestran el coeficiente significativo de la variable edad $(-0,06)$ sobre el rendimiento académico universitario. Además, el coeficiente de la variable producto EC*E, creada a partir del producto de la variable dummy evaluación continuada (EC) y la variable edad (E), no resultó significativo. Esto indica que los efectos negativos que el incremento de edad ejerce sobre el rendimiento académico universitario se observan independientemente del sistema de evaluación elegido por el estudiante. A la vista de estos resultados no hay evidencia para rechazar la hipótesis $\mathrm{H} 2$ que defiende que la relación entre el incremento en la edad y el rendimiento académico universitario es independiente del sistema de evaluación adoptado en el curso de adaptación.

\section{CONCLUSIONES}

El proceso emprendido por las universidades europeas, con la declaración de Bolonia, para la construcción del EEES, hace que el paso por la universidad vuelva a ser considerado por diferentes segmentos demográficos de la sociedad. De este modo el perfil de estudiantes que accede a la universidad, proveniente de la etapa educativa inmediatamente anterior y que ha seguido ininterrumpidamente el itinerario temporal e institucional del proceso educativo hasta incorporarse al ámbito universitario se amplía, dando paso a estudiantes de mayor edad generando un panorama más heterogéneo en el aula.

En este contexto de diversidad el trabajo se ha enfocado hacia el análisis de la relación entre la edad del estudiante universitario y su rendimiento académico universitario, tomando como referencia una asignatura de finanzas, de carácter presencial de un curso de adaptación al Grado de $\mathrm{ADE}$, que por su naturaleza, dirigido a titulados de la anterior ordenación, posibilita la creación de grupos de población heterogénea. En este sentido, nuestro estudio extiende y completa otros análisis realizados sobre muestras heterogéneas respecto a la edad (Jiménez-Fernández, 1987; Cheung y Kan, 2002), pero referidos a programas de enseñanza a distancia y no presencial, en los que se ofrece cierta flexibilidad a los estudiantes para que puedan adaptar su estructura a sus propias necesidades. Además, en el estudio analizamos si los efectos de la edad sobre el rendimiento podrían diferir en dos contextos distintos de evaluación de la asignatura, un sistema de evaluación continuada y un sistema de evaluación global.

En general, en el período analizado (curso académico 20112012), los estudiantes matriculados en la asignatura del curso de adaptación al Grado se han mostrado favorables a participar en el método de evaluación continuada propuesto en la asignatura. No se observa que la preferencia por dicho sistema, frente a un sistema global, difiera en función de la edad del estudiante. Los resultados de los análisis muestran que tanto la edad, como el sistema de evaluación elegido por el estudiante son factores que influyen sobre su rendimiento académico universitario, aproximado éste por la tasa de rendimiento de la asignatura, en la primera convocatoria del semestre en la que se ubica dentro del curso de adaptación. Los resultados relativos a la efectividad del sistema de evaluación continuada sobre el rendimiento muestran, para el período analizado, que optar a un sistema de evaluación continuada, frente a uno global, tiene efectos positivos sobre el rendimiento. Estos resultados están en línea con los de estudios previos que, tanto a nivel nacional como internacional, han comprobado los efectos positivos que el sistema de evaluación continuada ejerce sobre el rendimiento (Coll et al., 2007; Clavería, 2009; Fraile et al., 2013).

Atendiendo a las hipótesis planteadas se comprueba que el rendimiento académico universitario disminuye a medida que aumenta la edad del estudiante, mostrándose, además, que dichos efectos se mantienen independientemente de haber optado por un sistema de evaluación continuada o un sistema de evaluación global. Estos resultados extienden los de estudios previos realizados en esta línea (Porto y Di Gresia, 2004; Cortés y Palomar, 2008), demostrando que el rendimiento difiere según la edad del estudiante, siendo más notable para los estudiantes más jóvenes. Además, nuestros resultados refuerzan la evidencia empírica previa, ya que el efecto negativo de la edad sobre el rendimiento universitario se observa independientemente del sistema de evaluación aplicado.

Una de las limitaciones del estudio se basa en la no consideración de variables de naturaleza psicológica como la habilidad social, el autocontrol, la motivación, etc., que 
pueden estar afectando al rendimiento académico universitario. Las variables psicológicas y pedagógicas también pueden determinar el rendimiento, así que recoger información sobre ellas e incluirlas como variables de control en los modelos analizados podría reforzar las conclusiones obtenidas. Las conclusiones del presente trabajo ponen de manifiesto la necesidad de desarrollar mecanismos de enseñanza aprendizaje que permitan a los estudiantes compatibilizar sus estudios con las responsabilidades familiares y laborales que generalmente acompañan al perfil de estudiantes de mayor edad, así como la necesidad de reforzar la adquisición de habilidades tecnológicas que pueden facilitar su aprendizaje. Las necesidades mencionadas se plantean especialmente relevantes en un curso de adaptación al grado, donde la distancia en el tiempo de finalización de los estudios acompaña las mayores responsabilidades familiares y laborales que pueden llegar a compartirse en esta etapa de reencuentro con la vida universitaria.

\section{REFERENCIAS}

Allingham, M. (2002). Choice Theory: A very short introduction, Oxford Press.

Becker, G. (1964). Human Capital, Columbia University Press NBER.

Byrne, M., y Flood, B. (2008). Examining the relationships among background variables and academic performance of first year accounting students at an Irish University. Journal of Accounting Education, 26(4), 202-212.

Clavería González, O. (2009). ¿Puede ayudar la evaluación continua a mejorar el rendimiento de los alumnos?. Revista de Formación e Innovación Educativa Universitaria, 2(2), 60-75.

Coll C., Rochera, M.J., Mayordomo, R.M. y Naranjo, M. (2007). Evaluación continua y ayuda al aprendizaje. Análisis de una experiencia de innovación en educación superior con apoyo de las TIC. Electronic Journal of Research in Educational Psychology, 5(13), 783-804.

Cortés Flores, A. y Palomar Lever, J. (2008). El proceso de admisión como predictor del rendimiento académico en la educación superior. Universitas Psychologica, 7(1), 197-213.

Cheung, L.L. y Kan, A. C. (2002). Evaluation of factors related to student performance in a distance-learning business communication course. Journal of Education for Business, 77(5), 257-263.

Daugherty, K., Ceballos-Coronel, M.L. y Soja, W. (2015). Sensitivity and specificity of course grades after exam failure used as an indicator for final course performance. Currents in Pharmacy Teaching and Learning, 7(2), 163-168.

Fraile, A., López, V.M., Castejón, F.J. y Romero, R. (2013). La evaluación formativa en docencia universitaria y el rendimiento académico del alumnado. Aula abierta, 41(2), 23-34.

Guney, Y. (2009). Exogenous and endogenous factors impacting student performance in undergraduate accounting modules. Accounting Education: An International Journal, 18(1), 51-73.
Jiménez-Fernández, C. (1987). Rendimiento académico en la universidad a distancia: Un estudio empírico sobre su evolución y predicción. Revista de Educación, 284, 317347.

Justice, E. y Dornan, T. (2001). Metacognitive Differences between Traditional-Age and Nontraditional-Age College Students”. Adult Education Quarterly, 51(3), 236-224.

Leder, G. C. y Forgasz, H. J. (2004). Australian and international mature students: The daily challenges. Higher Education Research\& Development, 23, 183198.

Martínez, J.R. y Galán, F. (2000). Estrategias de aprendizaje, motivación y rendimiento académico en alumnos universitarios. Revista de Española de Orientación y Psicopedagogía, 11(19), 35-45.

Martínez Riza, F. (2013). Dificultades para implementar la evaluación formativa Revisión de literatura, Perfiles Educativos, 35(139, 128-150.

McKenzie, K. y Gow, K. (2004). Exploring the first year academic achievement of school leavers and mature-age students through structural equation modeling. Learning and Individual Differences, 14(2), 107-123.

Morgan, M. (2012). The context of learning in higher education, In M. Morgan. (Ed.), Improving the student experience: A practical guide for universities and colleges (cap. 1). Abingdon, Oxon: Routledge.

Peiperl, M y Trevelyan, R. (1997). Predictors of performance at business school and beyond: Demographic factors and the contrast between individual and group outcomes. Journal of Management Development, 16(5), 354-367.

Porto, A. y Di Gresia, L. (2004). Rendimiento de estudiantes universitarios y sus determinantes. Revista de Economía y Estadística, 42(1), 93-113.

Ramsay, S., Jones, E. y Barker, M. (2007). Relationship between adjustment and support types: Young and mature-aged local and international first year university students. Higher Education, 54(2), 247-265.

Schuezte, H. y Slowey, M. (2002). Participation and exclusion: A comparative analysis of non-traditional students and lifelong learners in higher education. Higher Education, 44, 309-327.

Tejedor, F. J. (2003). Poder explicativo de algunos determinantes del rendimiento en los estudios universitarios. Revista española de pedagogía, 61, 5-32.

Tejedor, F.J. y García-Valcárcel, A. (2007). Causas del bajo rendimiento del estudiante universitario (en opinión de los profesores y alumnos. Propuestas de mejora en el marco del EEES. Revista de Educación, 342(1), 443473.

Tinto, V. (1975). Dropout From Higher Education: A Theoretical Synthesis of Recent Research. Journal of Higher Education, 45, 89-125.

Tones, M., Fraser, J., Elder, R. y White, K. (2009). Supporting mature-aged students from a low socioeconomic background. Higher Education, 58(4), 505-529. 